\title{
Trends in Quality-Adjusted Life-Years Lost Contributed by Smoking and Obesity
}

\author{
Haomiao Jia, PhD, Erica I. Lubetkin, MD, MPH
}

\begin{abstract}
Background: Quality-adjusted life-years (QALYs) use preference-based measurements of healthrelated quality-of-life (HRQOL) to provide an assessment of the overall burden of disease using a single number.
\end{abstract}

Purpose: This study estimated QALYs lost contributed by smoking and obesity for U.S. adults from 1993 to 2008.

Methods: Population HRQOL data were from the 1993-2008 Behavioral Risk Factor Surveillance System. The QALYs lost contributed by a risk factor is the sum of QALYs lost due to morbidity in the current year and future QALYs lost in expected life-years due to premature deaths (mortality). Premature deaths were estimated from the National Health Interview Survey Linked Mortality Files and mortality statistics.

Results: From 1993 to 2008, the proportion of smokers among U.S. adults declined 18.5\% whereas the proportion of obese people increased $85 \%$. The smoking-related QALYs lost were relatively stable at 0.0438 QALYs lost per population. In 1993 the QALYs lost were much smaller for obesity compared to smoking, with obesity contributing about 0.0204 QALYs lost. However, as a result of the increasing prevalence of obesity, the contribution of obesity-related QALYs lost increased consistently and had increased by $127 \%$ in 2008 when obesity resulted in 0.0464 QALYs lost, slightly more than smoking did. Smoking had a bigger impact on mortality than morbidity, whereas obesity had a bigger impact on morbidity than mortality.

Conclusions: This study estimated the overall burden of smoking and obesity over time and results indicate that because of the marked increase in the proportion of obese people, obesity has become an equal, if not greater, contributor to the burden of disease than smoking. Such data are essential in setting targets for reducing modifiable health risks and eliminating health disparities.

(Am J Prev Med 2010;38(2):138 -144) @ 2010 American Journal of Preventive Medicine

\section{Introduction}

I $\mathrm{n}$ delineating the framework and format of Healthy People 2020, the Secretary's Advisory Committee recommended that the best possible information be assembled on selected criteria that would assist users in prioritizing each health objective. ${ }^{1}$ These criteria center

From the Department of Biostatistics (Jia), Mailman School of Public Health and School of Nursing, Columbia University; and Department of Community Health and Social Medicine (Lubetkin), Sophie Davis School of Biomedical Education at The City College of New York, New York, New York

Address correspondence and reprint requests to: Haomiao Jia, $\mathrm{PhD}$, Columbia University, 630 West 168th Street, New York NY 10032. E-mail: hj2198@columbia.edu.

The full text of this article is available via AJPM Online at www.ajpmonline.net.

0749-3797/00/\$17.00

doi: 10.1016/j.amepre.2009.09.043 on the overall burden of disease (associated with a particular risk factor, determinant, disease or injury), the degree to which the burden can be prevented or reduced, and the cost effectiveness of alternative opportunities to reduce the health burden. In analyzing the health impact of risk factors, mortality or morbidity statistics such as attributable mortality and health-related quality of life (HRQOL) commonly have been used as outcome measures. ${ }^{2}$ However, as noted by the Secretary's Advisory Committee, a single measurement such as the qualityadjusted life-year (QALY) would be particularly useful in quantifying the overall health impact of risk factors using one number. ${ }^{1,3}$

Quality-adjusted life-years use preference-based measurements of health-related quality of life (HQROL) to provide an assessment of the overall burden of diseases as- 
sociated with both mortality and morbidity. ${ }^{4}$ Preferencebased HRQOL measures use summary scores (i.e., utility values) to represent population preferences for different health states. Thus, 1 year of life lived at a utility score of 0.8 is equal to 0.8 QALY. The total QALYs lost contributed by a risk factor includes the QALYs lost due to nonfatal diseases (morbidity) in the current year and the future QALYs lost in the expected life-years as a result of premature deaths (mortality). ${ }^{5-7}$

Burden of disease and cost-effectiveness analyses are especially useful for quantifying the impact of particular modifiable risk factors, analyzing disparities in QALYs both at the national and local (community) levels and for small sociodemographic subgroups, and examining changes over time. However, prior to Year 2000 these analyses were not able to be conducted in the U.S. because of the lack of a data set that contained health utility scores for a representative sample of the population. During 2000 2003, the Medical Expenditure Panel Survey (MEPS) included the EQ-5D, a QALY-compatible and preferencebased instrument. ${ }^{8}$ Using EQ-5D scores from the 2000 MEPS, an estimate has been made of the burden of disease attributable to obesity/overweight and low income in the general U.S. population. ${ }^{5,6}$ However, because the MEPS included the EQ-5D between 2000 and 2003 only and was designed to provide information for the entire nation-and not for individual states or at the local level-trends over time and geographic variations were unable to be examined.

This study examined the trend of the health burden of smoking and obesity for U.S. adults from 1993 to 2008 using currently available population-based data. These two modifiable risk factors have the greatest impact on morbidity and mortality in the U.S. ${ }^{9,10}$ The total QALYs lost were the sum of the QALYs lost due to a decrease in HRQOL score (morbidity) and the future QALYs lost in the expected life-years due to premature deaths (mortality) contributed by these two modifiable risk factors. The proposed method also can be used for examining health disparities and evaluating progress at the national, state, and local levels with regard to the upcoming Healthy People 2020 health objectives.

\section{Materials and Methods}

\section{Data and Measurements}

Population HRQOL data were from the 1993-2008 Behavioral Risk Factor Surveillance System (BRFSS), the largest ongoing state-based health survey of U.S. adults. ${ }^{11,12}$ The BRFSS is a random-digit-dialed telephone survey that interviews non-institutionalized civilian adult residents aged $\geq 18$ years to collect health-related data at the state and substate levels and to track trends over time. ${ }^{11,12}$ In this analysis, $\mathrm{N}=3,590,540$; the annual $n$ ranged from 102,263 in 1993 to 406,749 in 2008.

Since 1993, the BRFSS has included a set of questions for recent poor health days to describe and track the overall physical and mental health of populations. ${ }^{13}$ These measures, called the Healthy Days, ask respondents about the number of physically unhealthy days, mentally unhealthy days, and days with activity limitation during the past 30 days. The CDC also recommends constructing a summary measure of overall unhealthy days by adding physically and mentally unhealthy days, with a logical maximum of 30 unhealthy days. ${ }^{14}$ Because of the absence of other temporal and spatial data that measure population HRQOL, the CDC has promoted the BRFSS as being capable of filling an important data void. ${ }^{12}$

However, because the numbers of poor health days are not preference-based measures of HRQOL, the measures cannot be used to calculate QALYs for burden of disease and costeffectiveness analyses directly. ${ }^{14,15}$ Recognizing this limitation, the HRQOL Surveillance Expert Panel convened by the CDC's Division of Adult and Community Health recommended calibrating HRQOL scores to preference-based measures. ${ }^{16} \mathrm{Sev}-$ eral studies have examined the possibility for a statistical crosswalk between the Healthy Days and utility scores of HRQOL to develop multivariable conversion tables for formulas to estimate utility scores from unhealthy days. ${ }^{14,15}$

A previously constructed formula was used here for estimating EQ-5D index scores from the Healthy Days measures to the BRFSS respondents. ${ }^{15}$ The respondents' EQ-5D scores were estimated based on their number of overall unhealthy days, self-rated health status, and age category. The validation studies demonstrated that the estimating model fit data well ${ }^{17}$ and the estimated EQ-5D index score had acceptable validity in terms of providing accurate estimated mean scores in different subgroups needed for calculating QALYs. ${ }^{15}$

The National Center for Health Statistics produced the National Health Interview Survey (NHIS) Linked Mortality Files by linking eligible adults in the NHIS data to the National Death Index to obtain mortality follow-up through December 31, 2002. ${ }^{18}$ The NHIS contains sociodemographics and health behavior questions similar to the BRFSS. The 1997-2000 cohort of the NHIS Linked Mortality File and the detailed mortality data compiled by the $\mathrm{CDC}^{19}$ were used to estimate premature deaths due to each risk factor.

The hazard ratios for smoking (current smokers versus not) and obesity (BMI $\geq 30 \mathrm{~kg} / \mathrm{m}^{2}$ vs $\left.18.5-<30 \mathrm{~kg} / \mathrm{m}^{2}\right)$ were estimated from the NHIS Linked Mortality File data. The smoking- and obesity-associated excess death rate in each 1 -year age interval was calculated as

$$
\Delta_{i}=\frac{\left(H_{i}-1\right) p_{i} m_{i}}{H_{i} p_{i}+\left(1-p_{i}\right)},
$$

where $H_{i}$ is the estimated hazard ratio at age $i, m_{i}$ is the mortality rate, and $p_{i}$ is the proportion of people at risk for the same age estimated from the BRFSS. 


\section{Quality-Adjusted Life Expectancy (QALE)}

The detailed mortality data (by age and gender) also were used to construct life tables (in 1-year intervals) to calculate life expectancy at age $i^{20}$ Let $A_{i}$ be the number of the population surviving to age $i$ and $m_{i}$ be the mortality rate for age $i$. Suppose those who died during the 1-year interval lived 0.5 year on average, the total Life Year $B_{i}$ between ages $i$ and $i+1$ is $\left(1-m_{i} / 2\right) A_{i}$. The life expectancy at age $i, L E_{i}$, is total life years above age $i$ divided by the number of the population surviving to age $i$ or $L E_{i}=\sum_{j \geq i} B_{j} / A_{i}$. Because the QALYs between ages $i$ and $i+1$ is $B_{i} x_{i}^{5,6}$, the QALE at age $i$ is

$$
Q A L E_{i}=\left(\sum_{j \geq i} B_{j} x_{j}\right) / A_{i},
$$

where $x_{j}$ is the mean EQ-5D index score for the age interval.

\section{Quality-Adjusted Life-Years}

The total QALYs lost contributed by a risk factor is the sum of the QALYs lost in 1 year due to a decrease in the HRQOL score (morbidity) and the future QALYs lost in the expected lifeyears due to premature deaths or mortality. ${ }^{5-8}$ This analysis used the proportion of the population to calculate QALYs, which can be converted to total QALYs by multiplying with the adult population in each year.

The QALYs lost due to morbidity contributed by a risk factor is defined as the potential annual QALYs that would be gained if those at risk had a mean EQ-5D index score that was equal to the score of the reference group:

$$
\text { morb }=\sum_{i}\left(x_{i}^{0}-x_{i}^{1}\right) p_{i},
$$

where $x_{i}^{0}$ and $x_{i}^{1}$ are the mean EQ-5D index scores for the age interval of the reference group and at-risk group, respectively.

The future QALYs lost in the expected life-years due to excess death is the product of excess deaths and the potential QALE that would be gained:

$$
\begin{aligned}
\text { mort }= & \sum_{i}\left(\Delta _ { i } \left(\sum _ { j \geq i } B _ { j } \left(x_{j}^{0}\right.\right.\right. \\
& \left.\left.\left.-x_{j}^{1}\right) p_{j}\right) / A_{i}\right) .
\end{aligned}
$$

The total QALYs lost is $Q A L Y^{L}=$ morb + mort. categories

${ }^{\mathrm{a} B M I}=18.5-<30 \mathrm{~kg} / \mathrm{m}^{2}$

${ }^{\mathrm{b}}$ Estimated from the BRFSS

\section{Results}

This analysis first examined both the mean EQ-5D index scores according to obesity and smoking status and the hazard ratio for these two risk factors to analyze the impact of obesity and smoking on HRQOL (morbidity) and premature deaths (mortality), respectively (Table 1). Mean EQ-5D scores were 0.05 points lower for obese people compared to nonobese people ( 0.833 vs 0.883 ), and such a difference was much greater (about 51\% higher) than the mean score difference between smokers and nonsmokers $(0.846$ vs 0.879 or 0.033 points difference). However, the estimated hazard ratio for smokers was more than twice the hazard ratio for obese people (2.789 vs 1.259), indicating a much larger impact on mortality from smoking than from obesity.

Next is the annual life expectancy and HRQOL scores for U.S. adults (Figure 1). Although the life expectancy of a person aged 18 years had increased consistently from 1993 to 2008 by approximately $3.5 \%$ (from 58.4 years to 60.4 years), the HRQOL of the U.S. adult population had declined during the same time period. Mean EQ-5D index scores decreased from 0.883 to 0.864 , or by approximately $2.2 \%$, and such a decline in EQ-5D scores was found in every age and gender subgroup. With the combination of a longer life expectancy but worsening

Table 1. Smoking- and obesity-associated mean EQ-5D scores and hazard ratio by age

\begin{tabular}{|crllll|}
\hline & \multicolumn{1}{l}{$\boldsymbol{n}$} & Smoke & Do not smoke & Obese & Not obese $^{\mathbf{a}}$ \\
\hline $\begin{array}{c}\text { Mean EQ-5D } \\
\text { Total }\end{array}$ & $3,552,823$ & 0.846 & 0.879 & 0.833 & 0.883 \\
Age (years) & & & & & \\
$18-24$ & 229,851 & 0.893 & 0.935 & 0.905 & 0.928 \\
$25-44$ & $1,215,613$ & 0.864 & 0.913 & 0.870 & 0.909 \\
$45-64$ & $1,275,544$ & 0.809 & 0.860 & 0.805 & 0.866 \\
$65-74$ & 461,015 & 0.799 & 0.831 & 0.782 & 0.841 \\
$\geq 75$ & 370,800 & 0.753 & 0.773 & 0.732 & 0.781 \\
Hazard ratio & & & & & \\
Total & 124,483 & 2.789 & - & 1.259 & - \\
Age (years) & & & & & \\
$18-24$ & 12,883 & 1.075 & - & 0.576 & - \\
$25-44$ & 51,712 & 2.269 & - & 1.348 & - \\
$45-64$ & 35,554 & 2.094 & - & 1.074 & - \\
$65-74$ & 12,967 & 2.325 & - & 1.241 & - \\
$\geq 75$ & 11,367 & 2.084 & - & 1.021 & - \\
\hline
\end{tabular}

'Estimated from the NHIS Linked Mortality File 
HRQOL scores, the quality-adjusted life expectancy for a person aged 18 years increased slightly from 50.9 years to 51.7 years, or by approximately $1.7 \%$.

The proportion of smokers among U.S. adults declined approximately $18.5 \%$, from $22.7 \%$ in 1993 to $18.5 \%$ in 2008 (Figure 2). However, most of the decline occurred after 2002. By contrast, the proportion of obese people increased greatly, from $14.5 \%$ to $26.7 \%$, or an $85 \%$ increase, and the increase was consistent during this time period. During these 16 years, the total QALYs lost contributed by smoking was relatively stable (standard deviance $=0.1 \%$ ), at an average of 0.0438 QALYs lost per adult population in each year or 16.0 days of healthy life lost per adult population. For obesity, the total QALYs lost was much smaller in 1993, at 0.0204 QALYs lost per adult population or 7.5 days of healthy life lost per adult population. This number was approximately $47 \%$ of the QALYs lost contributed by smoking. However, QALYs lost contributed by obesity increased by $127 \%$ and consistently increased with the increase in the percentage of obesity from 1993 to 2008. In 2008, obesity resulted in 0.0464 QALYs lost per adult population which slightly exceeded the number of QALYs lost due to smoking.

The final analysis examined the total QALYs lost due to morbidity and due to mortality separately (Figure 3). The greatest number of QALYs lost was due to smoking-related mortality whereas smoking-related morbidity accounted for the least QALYs lost. For smoking, the QALYs lost due to morbidity increased from 0.0083 QALYs lost per adult population in 1993 to 0.0105 QALYs lost per adult population in 2008 , or a

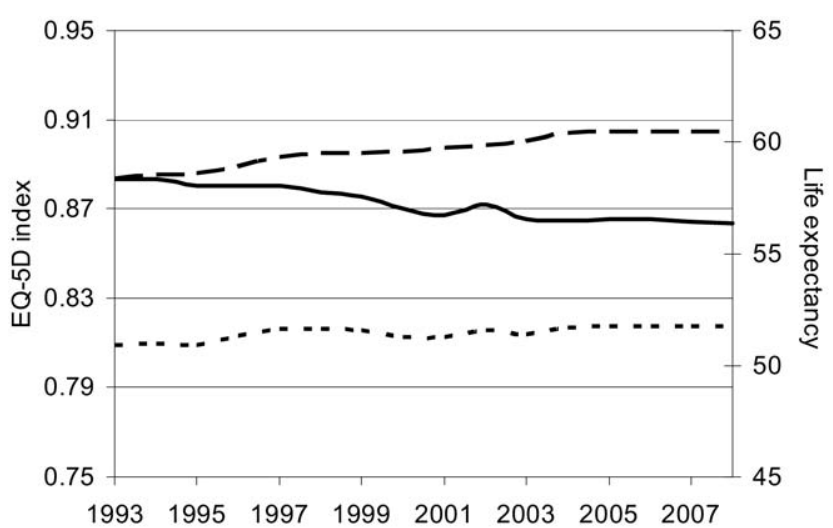

EQ-5D index - - LE at $18 \cdots$ QALE at 18

Figure 1. EQ-5D index, life expectancy at age 18 years (LE at 18), and quality-adjusted life expectancy at age 18 years (QALE 18) from 1993 to 2008

EQ-5D is a standardized instrument for use as a measure of health outcome. Applicable to a wide range of health conditions and treatments, it provides a simple descriptive profile and a single index value for health status.

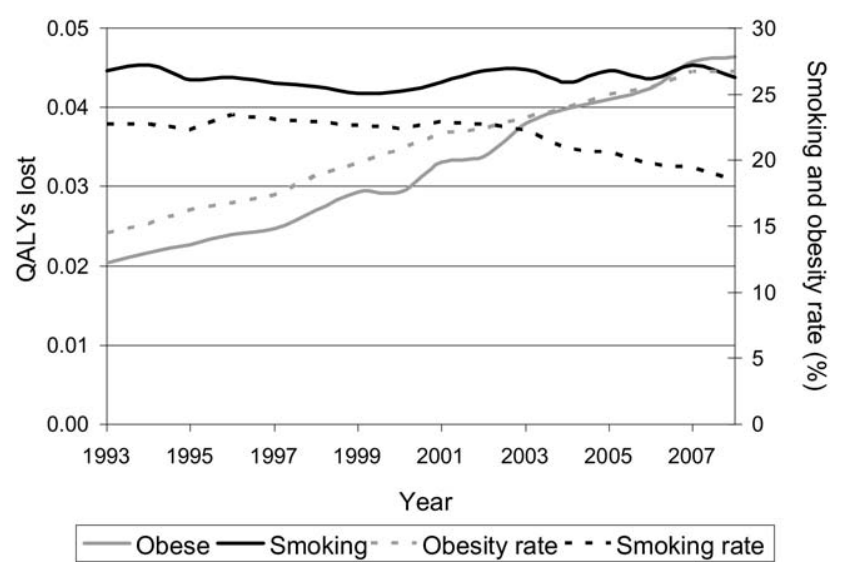

Figure 2. Quality-adjusted life-years lost per adult contributed by obesity and smoking, the obesity rate, and the smoking rate from 1993 to 2008

$26 \%$ increase, but the QALYs lost due to mortality decreased slightly from 0.0363 to 0.0333 QALYs lost per adult population, or an $8.4 \%$ decrease during this period. For obesity, both the QALYs lost due to mortality and that due to morbidity increased. The QALYs lost due to morbidity increased $141 \%$ (from 0.011 to 0.0265 QALYs lost per adult population), whereas the QALYs lost due to mortality increased $112 \%$ (from 0.0094 to 0.0199 QALYs lost per adult population). These results are consistent with the findings reported in Table 1 (i.e., smoking is associated with more premature deaths whereas obesity is associated with a greater negative impact on HRQOL).

\section{Discussion}

The overall health burden of obesity among U.S. adults has increased consistently since 1993 . The finding that

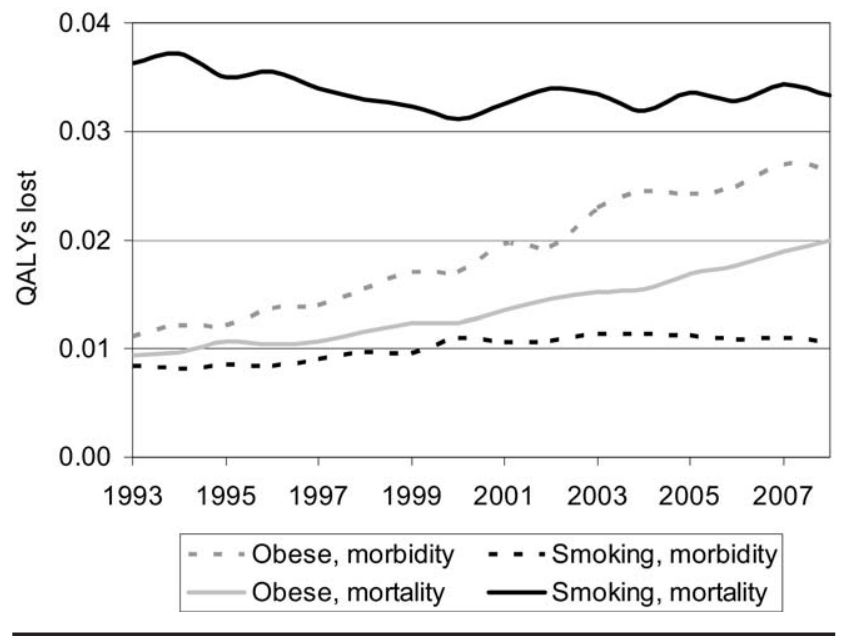

Figure 3. Quality-adjusted life-years lost per adult due to mortality and morbidity contributed by smoking and obesity from 1993 to 2008 
obesity is becoming an equal, if not greater, contributor to the burden of disease as smoking is consistent with the patterns noted in the profile of the leading causes of death. ${ }^{9,21,22}$ The substantial increase in the QALYs lost contributed by obesity was due to the dramatic increase in the prevalence of obesity in the U.S. ${ }^{23}$ By the final year examined (2008), the total health burden of obesity surpassed the total health impact of smoking.

Although the percentage of U.S. adults who are smokers has decreased since 2002, the burden contributed by smoking has remained stable. This may be due to the differences in peak prevalence of smoking and differences in susceptibility to smoking-associated conditions among subgroups of the population. ${ }^{24}$ Continued monitoring of the percentage of smokers and burden of disease is crucial, given the recent increase in the federal cigarette tax and the economic recession. ${ }^{25}$

Although life expectancy and QALE have increased over time, the increase in the contribution of mortality to QALYs lost from obesity may result in a decline in future life expectancy. ${ }^{26}$ With regard to HRQOL trends, HRQOL has declined during the period examined, and such a pattern is consistent with findings from other investigators in the U.S. and abroad. ${ }^{27-30}$ Indeed, the increasing contribution of morbidity to QALYs lost due to obesity may have accounted for a portion of the decline of HRQOL.

Previous analyses by the CDC had used the BRFSS data to estimate premature deaths due to smoking by calculating the smoking-attributable mortality and the years of potential life lost (YPLL) for the U.S. states and the District of Columbia from 2000 to $2004 .{ }^{31}$ The interpretation of YPLL from smoking is similar to the QALYs lost due to the mortality attributed to smoking, and these two measures are highly correlated $(r=0.81)$. Other analyses examined the decreases in HRQOL scores for smokers and obese people s2,33 $^{3,}$ as well as decreases in HRQOL scores due to diseases/conditions aligned with smoking and obesity, such as COPD and diabetes. ${ }^{34,35}$ Unlike these studies, a single measurement of QALYs was used in the current study for the overall health impact of smoking and obesity, thereby accounting for the effect on both mortality and morbidity.

Using a single value of QALYs to quantify the burden of disease enables the direct comparison of economic costs due to premature deaths and nonfatal disease contributed by a risk factor. With the calculation of QALYs lost due to morbidity and mortality, smoking has a greater burden from premature deaths than from disability and activity limitations, with the odds of the burden from mortality and morbidity being 3.4 to 1 . By contrast, obesity causes greater burden from disability and activity limitations than from premature deaths, and the odds due to morbidity and mortality has been approximately 1.3 to 1 .

There are several limitations in this analysis. First, the analysis relies on the validity of EQ-5D estimates from the Healthy Days measures. Different measures of HROQL may not reliably crosswalk among the full range of health states at the individual level. ${ }^{14,15}$ For example, because all model-based estimates tend to "regress toward the mean," this analysis might underestimate the total QALYs lost. Therefore, these calculations would likely undervalue the total health impact of smoking and obesity. However, estimated EQ-5D scores should have acceptable accuracy in terms of providing estimated mean scores in different subgroups needed for calculating QALYs. ${ }^{15,36}$ Second, the calculated CI of QALYs would be artificially too small and difficult to interpret because the scores used in this analysis were based on the model-estimated EQ-5D scores, not the actual observed EQ-5D scores. Also, because the model would underestimate EQ-5D scores of people with good health and overestimate scores for people with poor health, the estimated QALYs would be biased. Interpretation of CIs for biased estimates is difficult. ${ }^{37,38}$ Therefore, CIs and SEs are not reported. Third, in 2002, 28 states and the District of Columbia did not ask the Healthy Days questions. This may partially explain the outliers in the mean estimated EQ-5D index score in 2002. The estimated values for 2002 should be for only the 22 states that administrated these questions. Finally, BRFSS data are self-reported, and participants may have under- or over-estimated responses to certain items. Data show that participants tend to under-report both smoking and weight ${ }^{39,40}$ and, therefore, the burden of disease due to both smoking and obesity might actually be higher.

\section{Conclusion}

This study proposes a method that uses large, currently existing data sets representative of the U.S. general adult population to calculate QALYs lost contributed by two modifiable risk factors between 1993 and 2008. This method is well timed with the HRQOL Surveillance Expert Panel's goal that relates HRQOL surveillance to the Healthy People 2020 initiative. ${ }^{14}$ Resultant data might assist in the construction of specified quantitative targets for the Healthy People 2020 health objectives and setting priorities for prevention in a given population as well as according to sociodemographic subgroups. ${ }^{41}$ This method can be particularly useful when examining the health impact of some modifiable risk factors over time and at the local level (such as U.S. states and some substate areas) for burden of disease calculations and cost- 
effectiveness analyses. The proposed method takes the advantages of the richness of the BRFSS data for the tracking of population health outcomes and provides data that are necessary for examining the economic costs of modifiable risk factors. Although adding an intact, validated preference-based measure such as the EQ-5D to the Healthy Days measures might be a better long-term solution, trend data would not be available for a number of years.

No financial disclosures were reported by the authors of this paper.

\section{References}

1. The Secretary's Advisory Committee on National Health Promotion and Disease Prevention Objectives for 2020. Phase I report: Recommendations for the framework and format of Healthy People 2020. October 28, 2008. www.healthypeople. gov/hp2020/advisory/PhaseI/PhaseI.pdf.

2. Metzler M, Kanarek N, Highsmith K, et al. Community health status indicators project: the development of a national approach to community health. Prev Chronic Dis 2008;5(3):A94.

3. Field MJ, Gold MR, eds. IOM. Committee on Summary Measures of Population Health. Summarizing population health: directions for the development and application of population metrics. Washington DC: National Academies Press, 1998.

4. Gold MR, Siegel JE, Russell RB, Weinstein MC, eds. Costeffectiveness in health and medicine. New York: Oxford University Press, 1996.

5. Muennig P, Franks P, Jia H, Lubetkin E, Gold MR. The income-associated burden of disease in the U.S. Soc Sci Med 2005;61:2018-26.

6. Muennig P, Lubetkin E, Jia H, Franks P. Gender and the burden of disease attributable to obesity. Am J Public Health 2006;96:1662-8.

7. Sassi F. Calculating QALYs, comparing QALY and DALY calculations. Health Policy Plan 2006;21(5):402-8.

8. Clinical economics resource links: outcomes measurement. February 2005. Rockville MD: Agency for Healthcare Research and Quality. www.ahrq.gov/rice/ceoutc.htm.

9. Mokdad AH, Marks JS, Stroup DF, Gerberding JL. Actual causes of health in the U.S., 2000. JAMA 2004;291:1238-45.

10. Sturm R, Wells KB. Does obesity contribute as much to morbidity as poverty or smoking? Public Health 2001; 115:229-35.

11. Frazier EL, Franks AL, Sanderson LM. Using Behavioral Risk Factor Surveillance data. In: Using chronic disease data: a handbook for public health practitioners. Atlanta GA: CDC, 1992:4.1-4.17.

12. Mokdad AH, Stroup DF, Giles WH, Behavioral Risk Factor Surveillance team. Public health surveillance for behavioral risk factors in a changing environment. Recommendations from the Behavioral Risk Factor Surveillance team. MMWR Recomm Rep 2003;52(RR-9):1-12.

13. CDC. Measuring healthy days: population assessment of health-related quality of life. Atlanta GA: CDC, 2000.
14. Jia H, Lubetkin EI, Moriarty DG, Zack MM. A comparison of Healthy Days and EuroQol EQ-5D measures in two U.S. adult samples. Appl Res Qual Life 2007;2:209-21.

15. Jia H, Lubetkin EI. Estimating EuroQol EQ-5D scores from population Healthy Days data. Med Decis Making 2008; 8(4):491-9.

16. CDC. National Center for Chronic Disease Prevention and Health Promotion, Division of Adult and Community Health (DACH). Summary of the health-related quality of life (HRQOL) surveillance expert panel. Atlanta GA: CDC, 2008.

17. Jia H, Zack MM, Moriarty DG, Fryback DG. Predicting the EuroQol EQ-5D index from the CDC's Healthy Days measures in a national U.S. sample. Paper presented at the 26th Annual Behavioral Risk Factor Surveillance System (BRFSS) Conference; March 2009; Atlanta GA.

18. National Center for Health Statistics. National Health Interview Survey Linked Mortality Files. www.cdc.gov/nchs/r\&d/ nchs_datalinkage/nhis_data_linkage_mortality_activities. htm.

19. CDC. Compressed mortality file: underlying cause-of-death. wonder.cdc.gov/mortSQL.html.

20. Anderson RN. Method for constructing complete annual U.S. life tables. National Center for Health Statistics. Vital Health Stat 2(129), 1999.

21. McGinnis JM, Foege WH. Actual causes of death in the U.S. JAMA 1993;270(18):2207-12.

22. CDC. U.S. obesity trends 1985-2007. www.cdc.gov/nccdphp/ dnpa/obesity/trend/maps/.

23. Reuser M, Bonneux LG, Willekens FJ. Smoking kills, obesity disables: a multistate approach of the U.S. Health and Retirement Survey. Obesity (Silver Spring) 2009;17(4):783-9.

24. Meara ER, Richards S, Cutler DM. The gap gets bigger: changes in mortality and life expectancy, by education, 19812000. Health Aff (Millwood) 2008;27(2):350 - 60.

25. Barclay E. Outlook hazy for smoking rates in the U.S. Lancet 2009;373(9673):1415.

26. Olshansky SJ, Passaro DJ, Hershow RC, et al. A potential decline in life expectancy in the U.S. in the 21 st century. N Engl J Med 2005;352(11):1138-45.

27. Zack MM, Moriarty DG, Stroup DF, Ford ES, Mokdad AH. Worsening trends in adult health-related quality of life and self-rated health-U.S., 1993-2001. Public Health Rep 2004;119(5):493-505.

28. Martin LG, Schoeni RF, Freedman VA, Andreski P. Feeling better? Trends in general health status. J Gerontol B Psychol Sci Soc Sci 2007;62(1):S11-21.

29. Lakdawalla DN, Bhattacharya J, Goldman DP. Are the young becoming more disabled? Health Aff (Millwood) 2004;23(1):168-76.

30. Burström K, Johannesson M, Rehnberg C. Deteriorating health status in Stockholm 1998-2002: results from repeated population surveys using the EQ-5D. Qual Life Res 2007;16(9):1547-53.

31. CDC. State-specific smoking-attributable mortality and years of potential life lost-U.S., 2000-2004. MMWR Morb Mortal Wkly Rep 2009;58(2):29-33.

32. Jia H, Lubetkin EI. The impact of obesity on health-related quality-of-life in the general adult U.S. population. J Public Health 2005;27(2):156-64.

33. Strine TW, Okoro CA, Chapman DP, et al. Health-related quality of life and health risk behaviors among smokers. Am J Prev Med 2005; 28(2):182-7. 
34. Pickard AS, Wilke C, Jung E, Patel S, Stavem K, Lee TA. Use of a preference-based measure of health (EQ-5D) in COPD and asthma. Respir Med 2008;102(4):519-36.

35. Clarke PM, Hayes AJ, Glasziou PG, Scott R, Simes J, Keech Ac. Using the EQ-5D index score as a predictor of outcomes in patients with type 2 diabetes. Med Care 2009;47(1):61-8.

36. Palta M, Chen H-Y, Fryback DG. Error standard deviations of five health utility indexes across the range of health: implications for assessing responsiveness. Presented at International Society for Quality of Life Research (ISOQOL) Annual Meeting; 2008 Oct 22-25; Montevideo, Uruguay.

37. Ganiats TG, Miller CJ, Kaplan RM. Comparing the qualityadjusted life-year output of two treatment arms in a randomized trial. Med Care 1995;33(4):AS245-54.
38. Statistics Canada. Health status indicators based on vital statistics. www.statcan.gc.ca/pub/82-221-x/2007001/qualityqualite/4063855-eng.htm.

39. Wagenknecht LE, Burke GL, Perkins LL, Haley NJ, Friedman GD. Misclassification of smoking status in the CARDIA study: a comparison of self-report and serum cotinine levels. Am J Public Health 1992;82:33-6.

40. Gorber SC, Tremblay M, Moher D, Gorber B. A comparison of direct vs self-report measures for assessing height, weight and body mass index: a systematic review. Obes Rev 2007;8(4): 307-26.

41. Kindig DA, Asada Y, Booske B. A population health framework for setting national and state health goals. JAMA 2008;299:2081-3. 